3. Son RC, Chang JC, Choi JH. Primary hepatic amyloidosis: report of an unusual case presenting as a mass. Korean $J$ Radiol. 2011;12:382-5.

4. Buck FS, Koss MN. Hepatic amyloidosis: morphologic differences between systemic AL and AA types. Hum Pathol. 1991;22:904-7.

5. Monzawa S, Tsukamoto T, Omata K, Hosoda K, Araki T, Sugimura K. A case with primary amyloidosis of the liver and spleen: radiologic findings. Eur J Radiol. 2002; 41:237-41.

\title{
A rare ERCP finding in an elderly patient with recurrent pancreatitis
}

Anomalous pancreaticobiliary junction (APBJ) forming a long common channel is a rare congenital anomaly, with a prevalence of 3:100,000 as reported in one population based series from Japan and it is accompanied with biliary cysts in $50-80 \%$ of patients; it is classified according to findings by Koji et al ${ }^{1,2}$ (Figure 1-a). Herein we present a 69-year-old previously healthy female who presented with cholangitis and pancreatitis and diagnosed to have Komi Type III c2 APBJ on endoscopic retrograde cholangiopancreaticography (ERCP).

\section{Case Report}

A 69-year-old female was referred to our ERCP unit from surgery clinic after diagnosis of acute cholangitis and pancreatitis was made. The patient was previously asymptomatic and had no history of chronic medical illness. Transabdominal ultrasonography performed for the differential diagnosis of cholangitis and pancreatitis revealed dilatation both in the intrahepatic bile ducts and ductus hepaticus communis but the distal part of the common bile duct (CBD) could not be evaluated

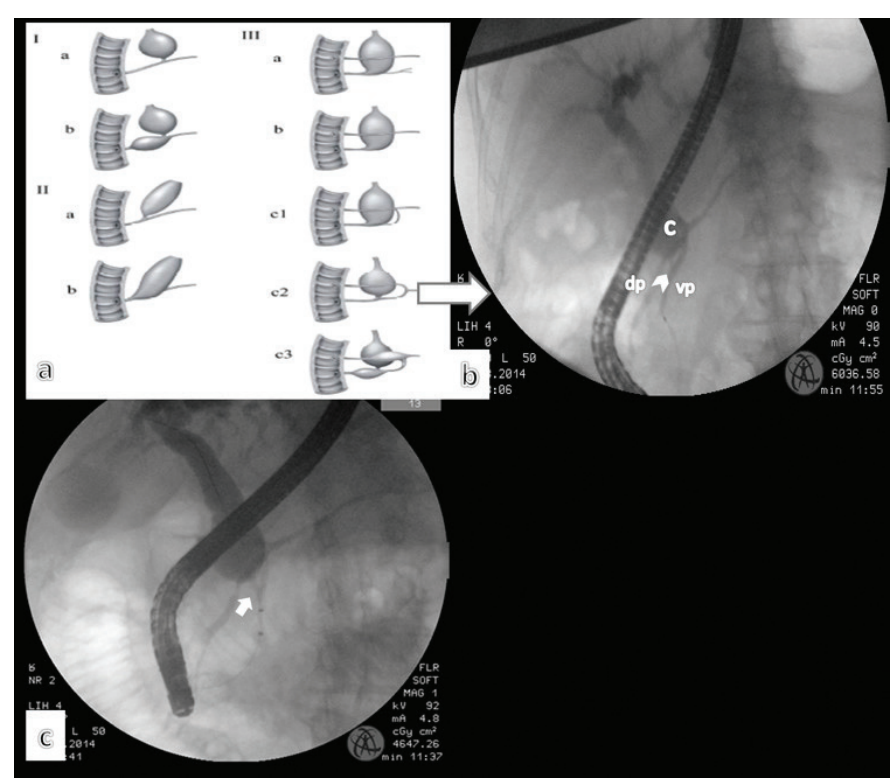

Figure-1: (a) Komi's classification; APBJ were classified into three types. The common bile duct joins the pancreatic duct at a right angle (Type I), the common bile duct joins the pancreatic duct at an acute angle (Type-II). In Type III, the common bile duct and the pancreatic ducts formed a complicated network. (b) Cholangiopancreatogram is compatible with Komi Type III c 2: Thin channel (broken arrow) which is located between choledocal cyst and ventral pancreatic duct (c, choledocal cyst; vp, ventral pancreatic duct; dp, dorsal pancreatic duct). (c) The guide wire is seen within the thin channel which is located between choledocal cyst and ventral pancreatic duct. (white arrow).

due to presence of gas in small intestine. The wall of the gallbladder was slightly thickened and there were small stones and sludge in the lumen. The pancreatic parenchyma was heterogenous. MR cholangiopancreaticography (MRCP) was performed which revealed dilatation of the intrahepatic bile ducts. The widest part of the CBD was $2 \mathrm{~cm}$ in diameter and it ended abruptly at the intrapancreatic level without any signs of intraluminal pathology. Both ventral and dorsal pancreatic ducts could be seen sepereately suggesting the presence of an incomplete pancreatas divisum. CEA and CA-19-9 levels were not elevated. By the third day of hospitalization, clinical symptoms and abnormal laboratory parameters improved rapidly and ERCP was performed based on the MRCP findings. ERCP revealed that the major papilla was located 
at third part of the duodenum, instead of the second. The major papilla could not be cannulated via guide wire as the wire repeatedly deviated towards the pancreatic duct and ultimately sphincterotomy was performed. Again, the wire had the tendency to deviate towards the pancreatic ductus, so pancreatogram was performed. When contrast medium was given, the presence of incomplete division was confirmed but surprisingly the biliary system was viewed as well. The distal part of the CBD recognized to have a cystic dilatation and was communicating perpendicularly with the ventral pancreatic duct via a thin channel. And thereafter it was opening to duodenum via non-dilated common long duct. (Figure-1b). Sphincterotomy of the major papilla was done. During ERCP it was observed that bile was present in minor papilla, so cholangiogram was also performed through it and sphincterotomy of the minor papilla was also done.

\section{Discussion}

According to Komi's classification of anomalous union between pancreatic and bile ducts, our patient was classified as a Type III-c2. ${ }^{2}$ One can speculate that the patient was asymptomatic throughout her life due to adequate drainage of pancreatic and biliary secretions via both the dorsal and the common long ducts and the obstruction of this complex network by a calculus or a protein plug originating from biliary system or pancreas precipitated her attack of acute pancreatitis and cholangitis.

This case suggests that this anomaly might be more common, some cases being asymptomatic, than previously estimated in general population. ERCP is the gold standard in the diagnosis of such pancreaticobiliary ductal variations and is the best method to obtain adequate information prior to any surgical intervention, as even MRCP had failed to document this variation in our case.

SELÇUK DİŞİBEYAZ ERKIN ÖZTAŞ UFUK BARIŞ KUZU NURETDIN SUNA ERTUĞRUL KAYAÇETIN
Department of Gastroenterology, Turkiye Yuksek Ihtisas Education and Research Hospital, Ankara, Turkey.

Correspondence: Ufuk Barlş Kuzu Email: ubarisk@gmail.com

\section{References}

1. Yamao K, Mizutani S, Nakazawa S et al. Prospective study of the detection of anomalous connections of pancreatobiliary ducts during routine medical examinations. Hepatogastroenterology. 1996;43(11):1238-45.

2. Komi N, Takehara H, Kunitomo K et al. Does the type of anomalous arrangement of pancreaticobiliary ducts influence the surgery and prognosis of choledochal cyst? J Pediatr Surg. 1992;27(6):728-31. 